\title{
Apply GNSS-Reflectometry Technique for wind retrieval in typhoon condition
}

\author{
Hwa Chien ${ }^{1}$, Quang-Huy Lu', Wen-Hao Yeh $^{2}$ \\ ${ }^{1}$ Institute of Hydrological \& Oceanic Sciences, National Central University, Taiwan \\ ${ }^{2}$ National Space Organization (NSPO), Taiwan
}

\begin{abstract}
Global Navigation Satellite System-Reflectometry (GNSS-R) is an innovative Earth observation technique that exploits signal from satellite constellations after reflection on the Earth surface. The GNSS-R techniques is also known to have the potential of mapping the surface wind speed up to $70 \mathrm{~m} / \mathrm{s}$, and thus provide a promising solution. Abundant real-time data of the typhoon surface wind speed will play the crucial role on the improvement of typhoon intensification forecasting. The aim of present study is to investigate the influences of high wind speed and the corresponding giant waves during typhoon to the GNSS-R wind speed retrieval algorithms. The Mean Square Slope that altered by the complex wave directionality is discussed. Finally, the uncertainties of wind speed retrieval with respect to the influences of wave directionality is assessed. The level 1 product from the GNSS-R receiver is a map of GPS signal power scattered from the sea surface, as a 2D function of delay and Doppler frequency, which is known as a Delay-Doppler Map, or DDM. Based on Zavorotny-Voronovich model, the DDMs are simulated from the Directional Mean Square Slope (DMSS) that obtained from the combination of capillary wave spectra and gravity wave spectra. The gravity wave spectra were calculated using a 3rd generation numerical wave model that driven by the Dujuan typhoon (2015) wind fields with super-fine resolution. The complexity of directional wave spectrum, such as extreme spatial heterogeneity, bimodal spectra and varying directional spreading alter the DMSS and DDM. Various observables, e.g. - DDM Average (DDMA), and Leading Edge Slope (LES) are then applied to the simulated DDM. Regression-based wind retrievals are developed for each individual observable using empirical geophysical model functions. The wind speed retrieval in case of Dujuan typhoon are compared with the target data uncertainty assessment.

Key word: GNSS-R, DDM, Typhoon, Sea Surface Wind speed, Z-V Model, Wave Directional Spectrum
\end{abstract}

\section{INTRODUCTION}

The GPS signal is reflected from the surface of the ocean, and processed to obtain information about the scattering surface. The receiver used to process these reflections essentially performs similar operations to those of an ordinary GPS receiver for positioning. The main difference is that the receiver deals with the signal scattered by the ocean surface, and not with a direct signal. The output of the processing also changes from a pseudo range measurement to a map of scattered power. For classical GPS positioning, the received signal is normally equal to the transmitted one, but delayed and shifted in frequency due to the Doppler Effect. For GNSS-R, the received signal is the result of the reflection of the incident GPS signal from the sea surface, which in principle distorts the incident signal according to its impulse response.

The final product from the GPS-R receiver is a map of GPS signal power scattered from the sea surface, as a $2 \mathrm{D}$ function of delay and Doppler frequency, which is known as a Delay-Doppler Map, or DDM. The generation of
DDMs from UK-DMC datasets has been carried out using the Daaxa software, developed by Scott Gleason [Gleason et al. (2005), Gleason (2006)]. Details of the software and the way it works can be found in [Gleason (2006)].

The estimation of sea state parameters is mainly based on the theory of wind-wave. So there is still a huge uncertainty estimating the sea state under very high wind speed condition, especially in Typhoon cases when the sea surface roughness changes dramatically. Successful acquisition of in situ wave measurements within a Tropical Cyclone (TC) is a difficult task. The conditions to be sampled, high winds and large waves, are energetic and often lead to the failure and destruction of instruments and platforms. Consequently, measurements of winds and waves in TCs remain rare.

Based on different quantities derived from the DDMs by NASA's Cyclone Global Navigation Satellite System (CYGNSS), five wind speed retrieval algorithms are developed. Each of these observable is introduced as a means of extracting wind speed information from the DDMs. Regression-based wind retrievals are developed for each individual observable using empirical geophysical model functions. The wind speed retrieval in case of Dujuan typhoon from those observables is 
compared to data from numerical model to verify the algorithm.

\section{THEORY}

\subsection{Sea Surface Roughness}

The mean square slope (MSS) of sea surface is defined as the variance of surface slope, which is a key parameter in understanding the air-sea interactions and ocean remote sensing. Air-sea fluxes of mass, momentum, and heat as well as radar scattering are believed to be significantly correlated with MSS. Many factors including wind speed, wave state, air- sea temperature difference, and intensity of sea surfactant films have impacts on the MSS.

The analysis of the source function balance leads to the conclusion that the dimensionless wavenumber spectrum, $\mathrm{B}(\mathrm{k})$, is functionally dependent on the dimensionless wind speed, $u_{*} / c$. Hwang and Wang in 2004 report an analysis of wave spectrum measurements in the ocean focusing on the wavenumber band between 1 and $300 \mathrm{rad} / \mathrm{m}$. This analysis produces a power-law function for $B\left(\frac{u_{*}}{c} ; k\right)$ :

$B\left(\frac{u_{*}}{c} ; k\right)=A(k)\left(\frac{u_{*}}{c}\right)^{a(k)}$

Because $\mathrm{c}$ and $\mathrm{k}$ are related by the wave dispersion relation $c^{2}=\left(\frac{g}{k}\right)+\tau k$ in deep water, where $\mathrm{g}$ is the gravitational acceleration and $\tau$ is the ratio of surface tension and fluid density, $B\left(\frac{u_{*}}{c} ; k\right)$ can be written as $B\left(u_{*} ; k\right)$, which is a convenient parameterization function of the ocean surface roughness spectrum.

The results of A and a are tabulated in Hwang (2005) as lookup tables to facilitate the computation of $B\left(u_{*} ; k\right)$. Hwang (2008) further simplifies the computational procedure by developing analytical functions for three wavenumber branches: $0<k \leq k_{1}, k_{1}<k \leq k_{2}$, and $k_{2}<k \leq \infty$, where $\mathrm{k} 1=1.5$ and $\mathrm{k} 2=300 \mathrm{rad} / \mathrm{m}$

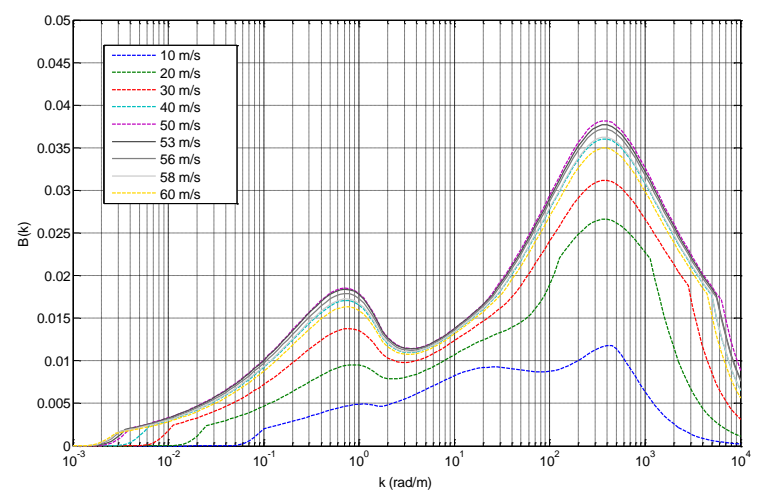

Figure 1. Wavenumber spectrum combined from low to highwind conditions, by Paul Hwang (RoughSpecPack2015) Figure 1 illustrated the variation of wavenumber spectrum over the large range of wavenumber coverage, calculated for different wind speed levels. The computed spectrum increases monotonically with U10 up to $50 \mathrm{~m} / \mathrm{s}$ and then decreases for $\mathrm{U} 10>50 \mathrm{~m} / \mathrm{s}$. It is recognized that there is still considerable uncertainty in the ocean surface drag coefficient in high wind.

An essential method to obtain the MSS is the integration of wave spectrum, which is defined as (Phillips 1977):

$s^{2}=\int_{0}^{\infty} k^{2} F(k) d k$

Where $F(k)$ is the wavenumber spectrum of ocean waves.

With the dispersion relationship of surface wave, equation (2) can be transformed into the integration of the frequency spectrum of the surface wave, $\mathrm{F}(\mathrm{f})$, which is the spectrum simulated using SWAN model. It can be rewritten as:

$s^{2}=\int_{0}^{\infty} \frac{(2 \pi f)^{4}}{g^{2}} F(f) d f$

Where $\mathrm{f}$ is the frequency.

The mean-square slope integrated from the spectral peak wavenumber $\mathrm{kp}$ to an arbitrary upper-bound wavenumber $\mathrm{ku}$ is denoted by:

$s^{2}\left(k_{u}\right)=\int_{k_{p}}^{k_{u}} k^{2} F(k) d k$

By varying U10 from 2 to $50 \mathrm{~m} / \mathrm{s}$ and $\mathrm{ku}$ from 1 to 104 $\mathrm{rad} / \mathrm{s}$, a dataset of $s^{2}\left(k_{u}\right)$ as a function of $\mathrm{U} 10$ and $\mathrm{ku}$ is created (Hwang 2013). The wind speed dependence deviates from the empirical linear relationship over the wide range of wind speeds and can be written as:

$s^{2}\left(k_{u}\right)=b_{2}\left(k_{u}\right) U_{10}^{2}+b_{1}\left(k_{u}\right) U_{10}+b_{0}\left(k_{u}\right)$

Least square fitting procedure is applied to compute $b_{2}\left(k_{u}\right), b_{1}\left(k_{u}\right)$, and $b_{0}\left(k_{u}\right)$ for wind sea and mixed sea conditions, as shown in Hwang 2013. Then, this above empirical equation can be used to calculate the MSS of an arbitrary wavenumber band of the ocean surface roughness.

\subsection{The Zavarotny-Voronovich Model for DDM Simulation}

The theoretical model commonly used to simulate DDMs, based on the theory formulated by [Zavorotny and Voronovich (2000)], and hereafter referred to as the Zavorotny-Voronovich (Z-V) model. This model describes the average scattering of GNSS signals from the sea surface, using a specific ElectroMagnetic (EM) approximation called Geometrical Optics (GO). The expression for the average scattered GPS power as a function of the delay and Doppler frequency, under the GO limit is the following:

$$
\begin{aligned}
T_{i}^{2} \iint_{S} \frac{1}{4 \pi R_{0}^{2} R_{T}^{2}} D^{2}(\boldsymbol{r}) \Lambda^{2}\left(\frac{\delta \tau}{\tau_{c}}\right) S^{2}\left(\frac{\delta f_{D}}{T_{i}}\right) \sigma_{o}(\boldsymbol{r}) d^{2} r
\end{aligned}
$$

where $\mathrm{S}$ is the scattering surface, and $\mathrm{r}$ is the point on the scattering surface; $\mathrm{Ti}$ is the coherent integration time (chosen equal to $1 \mathrm{~ms}$ for UK-DMC data); $1 / 4 \pi \mathrm{R} 20 \mathrm{R} 2 \mathrm{~T}$ represents the attenuation due to path losses, as R0 and RT are respectively the transmitter and receiver range from the scattering point; $\mathrm{D}(\mathrm{r})$ is the footprint function of the receiving antenna in terms of complex amplitudes, and D2 is the power antenna footprint; $\Lambda(\ldots) \cdot S(\ldots)$ is the Woodward Ambiguity Function or WAF $(\chi(\ldots))$ of pseudorandom C/A sequences; $\sigma 0$ (r) is the bistatic Normalized Radar Cross Section (NRCS). 
The NRCS is obtained using the GO approximation, resulting in the following mathematical expression:

$\sigma_{0}(\boldsymbol{r})=\frac{\pi|\Re|^{2} q^{4}}{q_{z}^{4}} f_{q}\left(\frac{-\boldsymbol{q}_{\perp}}{q_{z}}\right)$

It depends on the Fresnel reflection coefficient $\mathfrak{R}$, and on the Probability Density Function (PDF) fq () of the sea surface slopes, defined as $\mathrm{s}=-\mathrm{q} \perp / \mathrm{qz}=[-\mathrm{qx} / \mathrm{qz},-\mathrm{qy} / \mathrm{qz}]$. The vector $\mathrm{q}=[\mathrm{qx}, \mathrm{qy}, \mathrm{qz}]$ is known as the scattering vector, namely the vector obtained as the sum of the unitary vector pointing from the scattering point towards the transmitter, and the unitary vector pointing from the scattering point towards the receiver. This vector plays a crucial role, as it represents the ideal orientation that the tangent to the scattering point considered should have for the power to be scattered exactly towards the receiver.

Equation (7) for the NRCS does not specify a PDF of slopes and therefore is valid for any slope statistical distribution [Zavorotny and Voronovich (2000)]. In our case, we consider a gaussian PDF of the sea surface slopes, which also takes into account the wave direction [Germain et al. (2004)]. Its expression is as follows:

$f_{q}\left(\frac{-\boldsymbol{q}_{\perp}}{q_{z}}\right)=1 / 2 \pi \operatorname{det}(\boldsymbol{M}) \exp \left[-\left(\frac{1}{2} \boldsymbol{q}_{\perp}^{t} \boldsymbol{M}^{-1} \boldsymbol{q}_{\perp}\right)\right]$ $\mathbf{M}=$

$\left[\begin{array}{cc}\cos (\phi) & -\sin (\phi) \\ \sin (\phi) & \cos (\phi)\end{array}\right]\left[\begin{array}{cc}\sigma_{u p}^{2} & 0 \\ 0 & \sigma_{\text {cross }}^{2}\end{array}\right]\left[\begin{array}{cc}\cos (\phi) & \sin (\phi) \\ -\sin (\phi) & \cos (\phi)\end{array}\right]$ where $\sigma_{u p}^{2}$ and $\sigma_{\text {cross }}^{2}$ are respectively the Directional Mean Square Slopes (DMSS) along the major and minor axes of the 2D gaussian PDF, and $\varphi$ is the Principal Wave Slope Direction (PWSD), namely the angle between the $\mathrm{x}$-axis and the major axis of the PDF, clockwise. For brevity we call these two parameters the DMSS and PWSD. For wind generated waves, the DMSS can be thought as the MSS upwind and crosswind, and the PWSD as the angle defining the direction of the wind waves.

These three parameters are extremely important as they entirely characterize the Gaussian PDF of the sea surface slopes, and therefore they represent the sea surface roughness parameters in the $\mathrm{Z}-\mathrm{V}$ model. The $2 \mathrm{D}$ elliptical shape characterizing the Gaussian PDF reduces in amplitude for larger DMSS and decreases more slowly when moving away from the SP, consistent with the expected behavior of the scattered power for rougher seas. A rotation of such elliptical shape can be also noticed for changing $\phi$ values, indicating a change in the direction the waves are travelling.

\section{WIND SPEED RETRIEVAL - CONSIDER THE SWELL CONTRIBUTION TO DIRECTIONAL MSS}

The realistic ocean is the combination of wave types, especially in typhoon case. In which, swell contributes a very important affect to the totally ocean sea surface roughness. This chapter will verify the retrieved algorithm in case of the combination of mean square slope in both low and high frequencies. The approach detail is shown in Figure 2

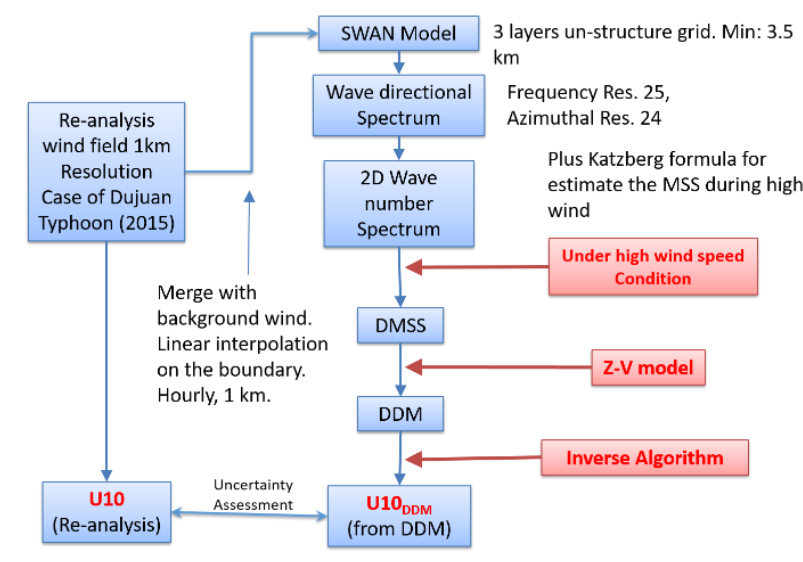

Figure 2. Wind speed retrieval approach - Consider the Swell contribution

\subsection{The fully range of MSS in frequency}

To verify the retrieved algorithm, the wave directional spectrum simulated from SWAN model using 3 layers unstructured grid has been used. The upwind and crosswind mean square slope, also the wave direction are calculated from spectrum that represented the ocean sea surface roughness effected by waves. For estimate the MSS during high wind, Katzberg formula has been added. The total MSS in full range of frequency is the combination of low and high wind MSS. The direction and MSS then are used as input for Z-V model to simulate DDM. The retrieved wind speed algorithm is applied to DDM to estimate wind speed using observables. After that, the retrieved wind speed will be compared to reanalysis wind field $1 \mathrm{~km}$ resolution (Figure 3 ) to estimate the uncertainty assessment.

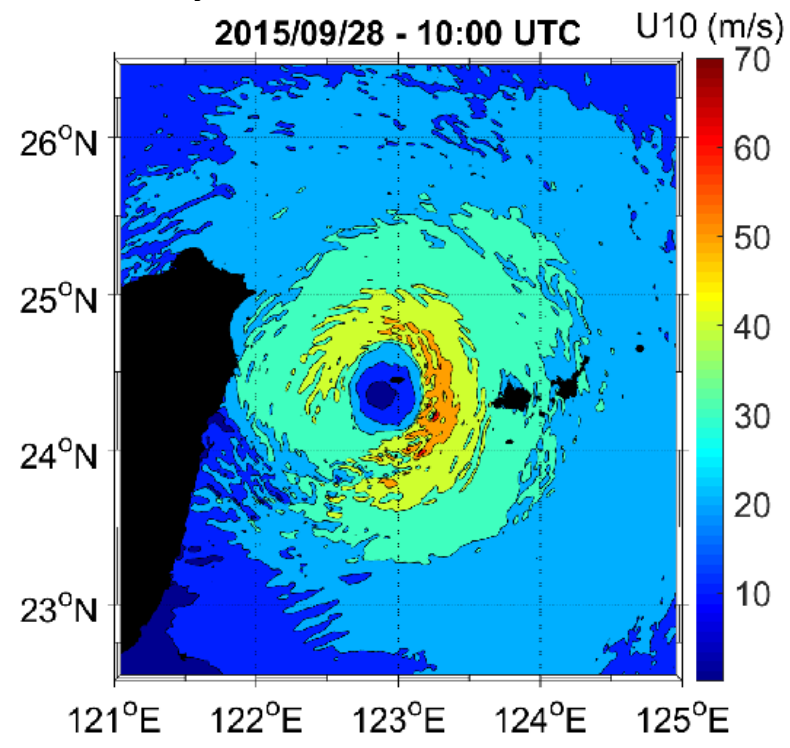

Figure 3. Wind field $1 \mathrm{~km}$ resolution $(10 \mathrm{~h}-2015 / 09 / 28)$ A method to obtain the MSS is the integration of wave spectrum, which is defined as (Phillips 1977):

$s^{2}=\int_{0}^{\infty} k^{2} F(k) d k$

Where $F(k)$ is the wavenumber spectrum of ocean waves. 
With the dispersion relationship of surface wave, equation (9) can be transformed into the integration of the frequency spectrum of the surface wave, $F(f)$, which is the spectrum simulated using SWAN model. It can be rewritten as:

$s^{2}=\int_{0}^{\infty} \frac{(2 \pi f)^{4}}{g^{2}} F(f) d f$

Where $\mathrm{f}$ is the frequency. DMSS can be calculated using (10), then combine to high frequency DMSS by Paul Hwang model to get the full range of MSS. The total MSS is shown in figure 4.
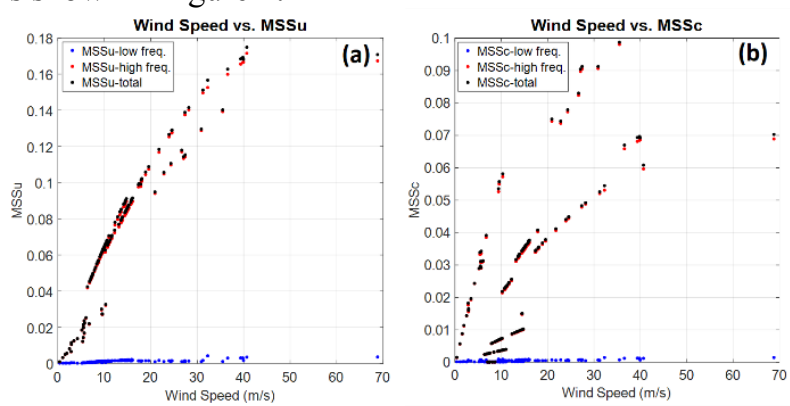

Figure 4. The variation of MSS upwind (a) and crosswind (b) in vertical axis with the increasing of wind speed (horizontal axis) Figure 4 shows the variation of MSS upwind and crosswind with the increasing of wind speed. The black dots represents total MSS, which is the combination of MSS in low and high frequencies. The development of DMSS during typhoon is in Figure 5. It shows the corresponding between MSS and wind field from typhoon eye to faraway, and between both sizes of the typhoon. The values of DMSS calculated by roughness spectrum are much higher than DMSS from wave model, which means effect of swell is considered in DMSS calculation. Those estimated DMSS then are used as the input parameter to simulate DDMs using Z-V model.
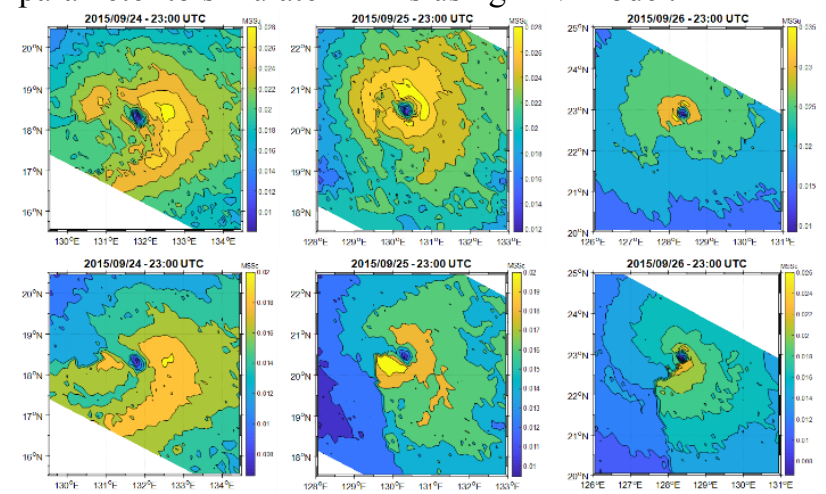

Figure 5. The variation of MSS during Dujuan typhoon

\subsection{Estimated Wind Speed and Uncertainty Assessment}

The direction extracted from wave spectrum and Directional Mean Square Slope in full range of frequency are used as the input parameter for $\mathrm{Z}-\mathrm{V}$ model to simulated Delay-Doppler Map (an example of Z-V DDM is shown in Figure 6).

A bulk of DDM have been simulated for 11204 data point over the domain, start from 6am 2015/09/23 to $7 \mathrm{pm}$
$2015 / 09 / 28$. The retrieved wind speed algorithm (mentioned in beginning of section 2.3) is applied to all those DDMs to estimate the wind speed during Dujuan typhoon time.
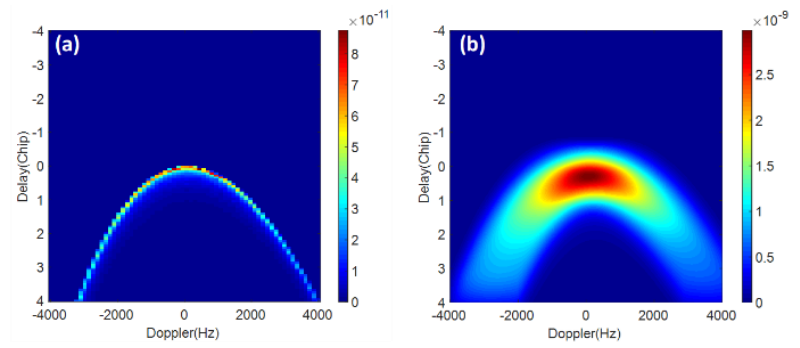

Figure 6. Simulated Z-V DDM in cases without Woodward Ambiguity Function (WAF) (a) and with WAF (b). The resolution is 128 (Delay) x 64 (Doppler frequency). Waves travelling assumed along the $\mathrm{x}$-direction $(\phi=0)$

At $23.980 \mathrm{~N}, 123.160 \mathrm{E}$, the wind speed at this point reaches to $69 \mathrm{~m} / \mathrm{s}$ in $10 \mathrm{~h}-2015 / 09 / 28$. Estimated wind speeds from both two observables, DDMA and TES are all match the reference wind speed. Root mean square error (RMSE) of estimated wind speed from DDMA is $1.4689 \mathrm{~m} / \mathrm{s}$ and RMSE of estimated wind speed from TES is $1.4018 \mathrm{~m} / \mathrm{s}$ which is quite acceptable. The highest retrieved wind speed at this location reach over $70 \mathrm{~m} / \mathrm{s}$ which mean the wind speed retrieve method is completely suitable for typhoon wind speed condition.

After applying GMFs for all 11204 data points, the estimated wind field has been re-constructed in simulated domain, then compare to re-analysis wind field $1 \mathrm{~km}$ resolution. The details can be seen in Figure 7, where the parallelogram domain represents the re-constructed wind field.
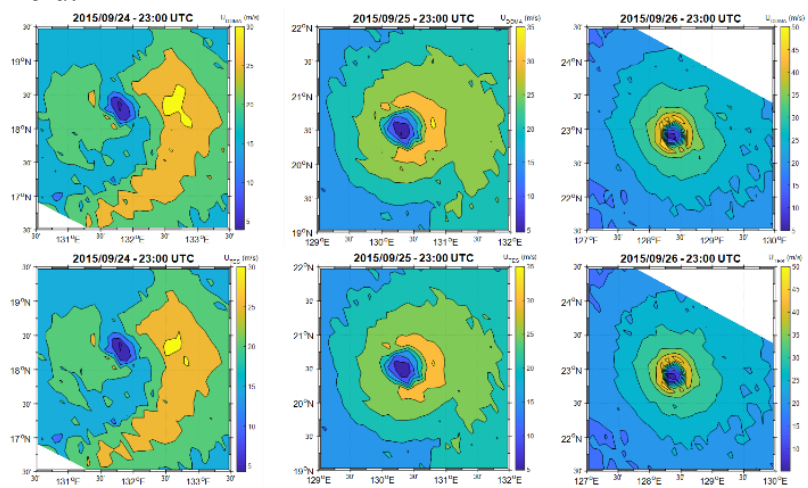

Figure 7. Comparison between 2 wind field

The uncertainties assessment between reference wind field and estimated wind is shown in figure 8 to visualize the range and other characteristics of responses for a large group. The central mark indicates the median; the bottom and the top edges of the box indicate the 25th and 75th percentiles, respectively. The whiskers extend to the most extreme data points not considered outliers, and the outliers are plot individually using the ' + ' symbol. Wind data is divided into six groups: $0-10 \mathrm{~m} / \mathrm{s}, 10-20 \mathrm{~m} / \mathrm{s}, 20$ $-30 \mathrm{~m} / \mathrm{s}, 30-40 \mathrm{~m} / \mathrm{s}, 40-50 \mathrm{~m} / \mathrm{s}$, and over $50 \mathrm{~m} / \mathrm{s}$. Group of $50-60 \mathrm{~m} / \mathrm{s}$ and $60-70 \mathrm{~m} / \mathrm{s}$ (and higher value) are merged to one group because of the limited number of samples. Each group is represented by the middle values 
of the groups $(7.04,13.59,24.2,35.49,45.60,59.66 \mathrm{~m} / \mathrm{s})$. When the wind increases, the deviation of estimated wind speed rises respectively. The box plot of the first 4 groups is comparatively short suggest that overall wind speed in those groups have a high level of agreement with each other. It means the combination DMSS method seems stable for low and middle range wind speeds. The box plot of group 5 is comparatively tall which means wind speed values in this group are diverse or maybe due to lack of data in this group and worthy further investigation in that group. However, the method also works quite well for high range of wind speed.
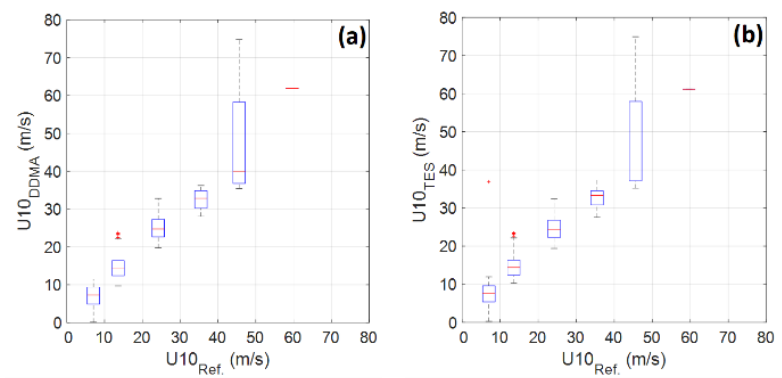

Figure 8. The uncertainties of retrieved wind speed using 2 observables DDMA (a) and TES (b) compared to reference wind speed (horizontal axis). On each box, the central mark indicates the median; the bottom and the top edges of the box indicate the 25 th and 75 th percentiles, respectively. The whiskers extend to the most extreme data points not considered outliers, and the outliers are plot individually using the ' + ' symbol.

\section{CONCLUSIONS}

The aim of the study is to assess the GNSS-R inverse algorithms for high wind speed conditions (storm condition). The $1 \mathrm{~km}$ re-analysised wind field of Dujuan Typhoon is used as input to drive the sea surface wave and roughness for DDM simulation. In the simulating case, as the retrieved wind speed reached to $70 \mathrm{~m} / \mathrm{s}$, the algorithm still works well. But real field data, especially at higher wind speed condition are needed for the validation.

Observables approach is processed. Two observables are derived from GNSS-R Delay-Doppler Maps (DDMs). Regression-based wind retrievals are developed for each individual observable using empirical geophysical model functions. The wind speed retrieval in case of Dujuan typhoon from those observables show good agreement with the data from numerical model. TES observable gives better results of wind speed retrieval than DDMA in idealized DDM.

The combination DMSS method that consider swell contribution can work in high wind condition, especially in typhoon to estimate wind speed. Further study is needed to test the statistic approach for real wind data. The expectation is to simulate the ocean wave surface based on roughness spectra in high wind, and then develop the algorithm with more realistic DDMs.

\section{REFERENCES}

[1] Antonio Rius et al, "Sea surface state measured using GPS reflected signals", GEOPHYSICAL RESEARCH LETTERS, VOL. 29, NO. 23, 2122 , doi:10.1029/2002GL015524, 2002.

[2] Attila Komjathy et al, "Retrieval of Ocean Surface Wind Speed and Wind Direction Using Reflected GPS Signals", American Meteorological Society 2004.

[3] B.Arguillat et al, "Measurements of the wavenumberfrequency spectrum of wall pressure fluctuations under turbulent flows", 11th AIAA/CEAS Aeroacoustics Conference (26th AIAA Aeroacoustics Conference) 23 - 25 May 2005, Monterey, California.

[4] Binglan Wang, Lili Song and Wenchao Chen, "Drag Coefficient during Strong Typhoons", Hindawi Publishing Corporation Advances in Meteorology Volume 2013, Article ID 650971.

[5] B. S. Gohil, Rajesh Sikhakolli, and Rishi Kumar Gangwar, "Development of Geophysical Model Functions for Oceansat-2 Scatterometer", IEEE GEOSCIENCE AND REMOTE SENSING LETTERS, VOL. 10, NO. 2, MARCH 2013.

[6] Charles Cox and Walter Munk, "Measurement of the Roughness of the Sea Surface from Photographs of the Sun's Glitter", JOURNAL OF THE OPTICAL SOCIETY OF AMERICA, VOLUME 44, NUMBER 11, NOVEMBER, 1954.

[7] Chen $\mathrm{Li}$ and Weimin Huang, "SIMULATING GNSS-R DELAY-DOPPLER MAP OF OIL SLICKED SEA SURFACES UNDER GENERAL SCENARIOS", Progress In Electromagnetics Research B, Vol. 48, 61-76, 2013.

[8] Chen Li and Weimin Huang, "An Algorithm for SeaSurface Wind Field Retrieval From GNSS-R DelayDoppler Map", IEEE GEOSCIENCE AND REMOTE SENSING LETTERS, VOL. 11, NO. 12, DECEMBER 2014.

[9] Christine P. Gommenginger, "TOWARDS LONGTERM SUSTAINABLE OBSERVATIONS OF OCEAN WIND AND WAVES WITH GNSS SIGNALS OF OPPORTUNITY".

[10] Donald E. Barrick, "Rough Surface Scattering Based on the Specular Point Theory", IEEE TRANSACTIONS ON ANTENNAS AND PROPAGATION, VOL. AP-16, NO. 4, JULY 1968.

[11] E. Cardellach et al, "GNSS-R ground-based and airborne campaigns for ocean, land, ice, and snow techniques: Application to the GOLD-RTR data sets", RADIO SCIENCE, VOL. 46, RS0C04, doi:10.1029/2011RS004683, 2011.

[12] Gary A. Mastin et al, "Fourier Synthesis of Ocean Scenes", 0272-1716/87/0300-0016-01.00 1987 IEEE.

[13] Giuseppe Foti et al, "Spaceborne GNSS reflectometry for ocean winds: First results from the UK TechDemoSat-1 mission", Geophysical Research Letters 10.1002/2015GL064204.

[14] Gary S. Brown et al, "The Wind-Speed Measurement Capability of Spaceborne Radar Altimeters", IEEE JOURNAL OF OCEANIC ENGINEERING, VOL. OE-6, NO. 2, APRIL 1981.

[15] Hasselmann et al, "Directional Wave Spectra Observed During JONSWAP 1973", Journal of Physical Oceanography.

[16] Hugo Carreno-Luengo et al, "GNSS-R Derived Centimetric Sea Topography: An Airborne Experiment Demonstration", IEEE JOURNAL OF SELECTED TOPICS IN APPLIED EARTH OBSERVATIONS AND REMOTE SENSING, VOL. 6, NO. 3, JUNE 2013. 
[17] H. Hersbach, "An improved C-band scatterometer ocean geophysical model function: CMOD5", JOURNAL OF GEOPHYSICAL RESEARCH, VOL. 112, C03006, doi:10.1029/2006JC003743, 2007.

[18] H. K. Johnson et al, "On the Dependence of Sea Surface Roughness on Wind Waves", JOURNAL OF PHYSICAL OCEANOGRAPHY, VOLUME 28.

[19] James L. Garrison et al, "Effect of sea roughness on bistatically scattered range coded signals from the Global Positioning System", GEOPHYSICAL RESEARCH LETTERS, VOL. 25, NO.13, PAGES 2257-2260, JULY 1, 1998.

[20] Juan Fernando Marchán-Hernández, “An Efficient Algorithm to the Simulation of Delay-Doppler Maps of Reflected Global Navigation Satellite System Signals", IEEE TRANSACTIONS ON GEOSCIENCE AND REMOTE SENSING, VOL. 47, NO. 8, AUGUST 2009. 